\title{
A Fast Algorithm for Computing Optimal Rectilinear Steiner Trees for Extremal Point Sets ${ }^{1}$
}

\author{
Siu-Wing Cheng ${ }^{2} \quad$ Chi-Keung Tang ${ }^{3}$ \\ Department of Computer Science \\ The Hong Kong University of Science and Technology \\ Clear Water Bay, Hong Kong
}

\begin{abstract}
We present an efficient algorithm to compute an optimal rectilinear Steiner tree for extremal point sets. A point set is extremal if every point in it lies on the boundary of a rectilinear convex hull of the point set. Our algorithm can be used to construct minimum-length connection for multi-terminal nets in homotopic routing in VLSI layout design. The previous best algorithms run in $O\left(k^{4} n\right)$ time and $O\left(n^{3}\right)$ time, where $n$ is the size of the point set and $k$ is the size of its rectilinear convex hull. Our algorithm runs in $O\left(k^{2} n\right)$ time which represents a significant improvement.
\end{abstract}

\footnotetext{
${ }^{1}$ This work is supported in part by the RGC grant HKUST 190/93E.

${ }^{2}$ E-mail: scheng@cs.ust.hk.

${ }^{3}$ E-mail: arion@cs.ust.hk
} 
Routing in VLSI layout design calls for connecting terminals in the same plane with horizontal and vertical wiring. The terminals are points that sit on the boundaries of isothetic modules which are impenetrable rectilinear polygons. So wiring can only be done in the routing region outside the modules. The terminals are divided into groups known as nets [13] and the terminals in each net are to be wired together. The connection within a net is a rectilinear Steiner tree (RST). A RST of a terminal point set $S$ is a tree with horizontal and vertical edges that spans all terminal points in $S$. Any tree vertex not in $S$ with degree at least three is a Steiner point. The total length of wiring is often required to be minimized which corresponds to finding a minimal (optimal) rectilinear Steiner tree (MRST). The general MRST problem is known to be NP-complete [7]. In homotopic routing [10], the user supplies a sketch of the wiring of the nets which specifies the placement of the modules and the topology of the wiring, but the wires are considered flexible. The problem is to determine the feasibility of the sketch and if feasible, compute the actual positions of the wiring so as to minimize the total wire length. In [9], it is shown that the sketch can be used to automatically partition the routing region outside the modules into rectilinear polygons without holes, such that each polygon contains exactly one net and the connection for each net must lie within its polygon. Thus, homotopic routing requires the solution to a special MRST problem where the terminal points lie on the boundary of a rectilinear polygon. It is commonly assumed that the input specifies the polygon and the terminal points in cyclic order on the boundary of this polygon [4, 12]. This paper describes an efficient algorithm when the terminal points lie on their rectilinear convex hull.

Previous results. Let $n$ be the size of $S$. Rectangle is the simplest rectilinear polygon. Aho et al. [2] are the first to present an $O\left(n^{3}\right)$-time algorithm for this case. Subsequently, this has been improved to $O(n)$ time by Cohoon $e$ al [5] and Agarwal and Shing [1]. Provan [11], Bern [3], and Erickson et al [6] propose algorithms for computing optimal graphical Steiner tree [8] for vertices on the outer boundary of a planar graph. Thus, their algorithms work for general rectilinear polygons. Provan's algorithm runs in $O\left(n^{6}\right)$ time and the algorithms by Bern [3] and Erickson et al [6] run in $O\left(n^{5}\right)$ time. When the planar graph is actually a grid graph (which is the case in our problem), Kaufmann et al [9] present an algorithm that runs in $O\left(n^{4} \log n\right)$ time in the worst case. Given the large number of nets that need to connected, a faster algorithm is desired. For the special case where the terminal points in $S$ lie on the boundary of a rectilinear convex hull of $S$, Richards and Salowe [12] present an $O\left(k^{4} n\right)$-time algorithm, where $k$ is the size of the rectilinear convex hull. When $k=O(1)$, the algorithm runs in linear time. But the running time degrades to $O\left(n^{5}\right)$ when $k=\Omega(n)$. Cheng et al [4] improve upon the worst-case and give an $O\left(n^{3}\right)$-time algorithm.

Our result. We present a dynamic programming $O\left(k^{2} n\right)$-time algorithm for the MRST problem when the terminals points in $S$ lie on the boundary of a rectilinear convex hull of $S$. Our main contribution is the identification of new structures in a MRST (e.g., common substructures, gaps, and divisions) which lead to a faster algorithm. Section 2 gives the basic definitions. Section 3 presents the basic ideas in our algorithm. Section 4 defines the partial solutions needed in dynamic programming. Sections $5-8$ provides a more in-depth discussion of the algorithm, proofs of correctness, and time complexity analysis.

\section{Definitions}

This section provides the definitions of and necessary concepts about rectilinear convex hull, extremal point set, grid graph, and rectilinear Steiner tree. All geometric objects in this paper are rectilinear (i.e., consists of horizontal and vertical line segments). For brevity, we omit 'rectilinear' for the rest of this paper. We also assume throughout this paper that $S$ is the input point set.

Convex hull and extremal point set. A shortest path between two points consists of an alternating sequence of horizontal and vertical edges such that both the $x$-coordinate and $y$-coordinate are monotonically non-decreasing or non-increasing (i.e., the path has the shape of a staircase). A polygon is convex if for any two points in it, there is a shortest path between them that lies within the polygon. Given a terminal point set $S$, a convex hull of $S$ is a smallest-area convex polygon containing $S$. We denote the boundary of the convex hull specified in the input by $R C(S)$. $R C(S)$ consists of four boundary staircases $B_{i}, 1 \leq i \leq 4$, which can be obtained by cutting $R C(S)$ at the highest, lowest, leftmost, and rightmost vertices. See Figure 1(a). For simplicity, we assume throughout this paper that $R C(S)$ has no 'pinched' vertex. See Figure 1(b) for a convex hull with pinched vertices. This assumption can be removed by an $O(n)$-time preprocessing [12]. An inner boundary corner is a reflex corner on $R C(S)$. An outer boundary corner is a convex corner on $R C(S)$. Note that an outer boundary corner vertex must be a terminal point. A boundary edge is a maximal straight line segment on $R C(S)$. $S$ is extremal if every terminal point in $S$ lies on $R C(S)$. 


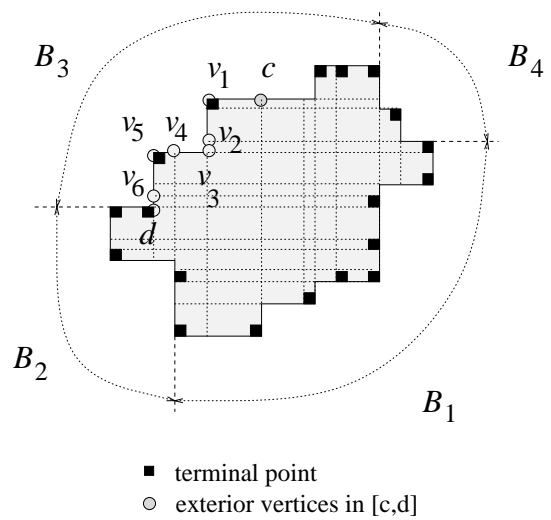

(a)

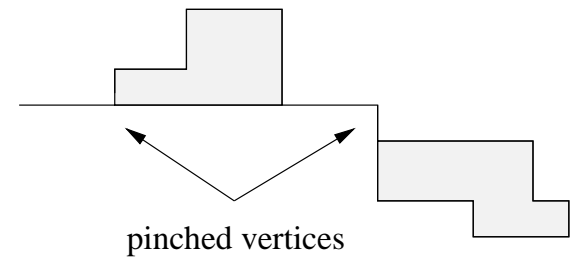

(b)

Figure 1:

Grid graph. A node is either a terminal point or the vertex at an inner boundary corner. A grid line is a line segment with endpoints on $R C(S)$ such that its interior lies inside $R C(S)$ and at least one endpoint is a node. The two endpoints of a grid line are called exterior vertices. Note that a node is an exterior vertex but not necessarily vice versa. A grid graph is the union of $R C(S)$ and all possible horizontal and vertical grid lines. The intersection of two grid lines inside $R C(S)$ is a grid vertex. Given exterior vertices $c$ and $d, B(c, d)$ is the portion of $R C(S)$ traversed from $c$ to $d$ in the counterclockwise order. Define $[c, d]$ to be the counterclockwisely ordered sequence of exterior vertices on $B(c, d)$. Define $S[c, d]$ to be subsequence of $[c, d]$ that contains $c, d$, and all terminal points in $[c, d]$. See Figure $1($ a) for an example: $[c, d]=\left\{c, v_{1}, v_{2}, v_{3}, v_{4}, v_{5}, v_{6}, d\right\}$ and $S[c, d]=\left\{c, v_{1}, v_{5}, d\right\}$. Note that for any exterior vertices $c$ and $d, S[c, d]$ is extremal.

Steiner tree. A Steiner tree vertex is either a terminal point, the intersection of two orthogonal line segments contained in the tree, or the vertex at an inner boundary corner. A tree edge is a line segment in the tree incident to two adjacent vertices. A Steiner point is a vertex that is not a terminal point and has degree at least three. An interior edge is a tree edge whose interior lies inside $R C(S)$. An interior line is a maximal sequence of adjacent and collinear interior edges. A complete interior line is an interior line whose endpoints lie on $R C(S)$. If $c$ and $d$ are endpoints of an interior line, then we denote the interior line by $(c, d)$ or $(d, c)$. A degree two vertex incident to two orthogonal tree edges is a corner-vertex. An interior corner is a corner whose corner-vertex lies inside $R C(S)$. The two interior lines incident to an interior corner-vertex are the legs of the interior corner. If both legs of an interior corner intersect $R C(S)$, it is called a complete interior corner. Three interior edges incident to the same vertex form a $\mathrm{T}$ and the vertex is a $\mathrm{T}$-vertex. The interior line that contains the two collinear interior edges is the head of the $\mathrm{T}$ or T-head. The interior line containing the other interior edge is the body of the T or T-body. Yang and Wing [14] prove that there is an optimal Steiner tree which is a subgraph of the grid graph. Therefore, we will assume that all Steiner trees considered in this paper are subgraphs of the grid graph. For any exterior vertices $c$ and $d$, whenever we refer to any 'interior edge/line/corner' of a Steiner tree for $S[c, d]$, we always refer to the interior of $R C(S)$ (not $R C(S[c, d])$ ). Let $\tau(S[c, d])$ be the set of all Steiner trees for $S[c, d]$ which are subgraphs of the grid graph.

\section{Basic ideas}

For each inner boundary corner of $R C(S)$, we shall assign a blue color to the two grid lines incident to the corner vertex and a constant number of grid lines nearby. Thus, we shall get $O(k)$ blue grid lines, where $k$ is the size of $R C(S)$. A line/edge is blue if it lies on a blue grid line. A complete interior corner is blue if its two legs are blue. An exterior vertex is blue it is the endpoint of a blue grid line or it is the vertex at an outer boundary corner. Our coloring procedure is an extension of that in [12]. In [12], for each inner boundary corner, the corner vertex and a constant number of terminal points are colored blue. Our procedure looks at the same set of terminal points for each inner boundary corner, but we color the grid lines incident to the corner vertex and these terminal points blue. Thus, our set of blue exterior vertices is a superset of that in [12]. This does not affect the properties of MRST proved in [12]. Due to lack of space, we omit the details of the color assignment in [12] as our algorithm does not depend on it. We want to point out that grid lines are colored blue only once by inspecting $R C(S)$. We may refer to the colors of vertices/edges/lines of a Steiner tree in $\tau(S[c, d])$ for some exterior vertices $c$ and $d$. Nevertheless, the coloring is always 
Steiner tree in $\tau(S[c, d])$. Since all vertices at the corners of $R C(S)$ are blue, we have the following useful fact:

Fact: Let $c$ and $d$ be exterior vertices such that $[c, d]$ contains a blue exterior vertex. Let $v$ be the blue exterior vertex in the sequence $[c, d]$ closest to $c$. Then $c$ and $v$ are contained in the same boundary edge.
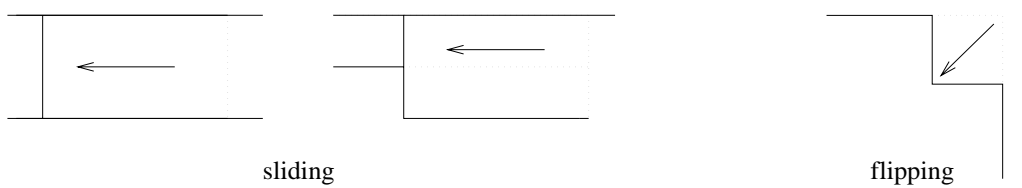

Figure 2:

It is proved in [12] that by sliding interior lines and flipping interior corners (Figure 2), there exists a subset of optimal Steiner trees in $\tau(S)$ that satisfy what we call the blue topological properties :

\section{Blue topological properties:}

B1 If $v_{1}$ and $v_{2}$ are two adjacent Steiner points in the interior of an interior line $\ell$, then both $v_{1}$ and $v_{2}$ have degree three. Moreover, the two interior lines properly incident ${ }^{4}$ to $\ell$ at $v_{1}$ and $v_{2}$ must be on opposite sides of $\ell$. That is, the interior lines properly incident to $\ell$ alternate between the two sides of $\ell$.

B2 The body of a $\mathrm{T}$ is an interior edge (i.e., it contains no Steiner point in its interior) and it is incident to a node on the boundary.

B3 Every interior corner must be a complete interior corner. Moreover, at most one of the two legs can have more than one interior edge properly incident to it. Each such properly incident edge is a T-body and is incident to a node on the boundary by B2. Among the interior edges properly incident to a leg $\ell$, the interior edge closest to the corner-vertex must lie on the side of $\ell$ opposite to the side of $\ell$ containing the other leg.

B4 No complete interior corner can be flipped to increase overlapping with the boundary, i.e., $R C(S)$.

B5 All legs of complete interior corners are blue. Among all interior edges properly incident to a leg, the one closest to the corner-vertex is blue.

B6 All complete interior lines whose interior contain Steiner point(s) are blue.

The blue topological properties imply that every interior line is either a complete interior line, a leg of a complete interior corner, or the body of a T. We define three groups of Steiner trees for $S$ that satisfy the blue topological properties. Group 1 contains no interior edge. Group 2 contains some complete interior corners. Group 3 contains some complete interior line but no complete interior corner. Thus, it suffices to compute a Steiner tree $G_{j}$ with total length no greater than the minimal tree in Group $j, 1 \leq j \leq 3$. (Computing the minimal tree in Group 3 directly is difficult as it is hard (and unnecessary) to guarantee that there is no complete interior corner.) The minimal tree among $G_{1}, G_{2}, G_{3}$ is the desired solution.

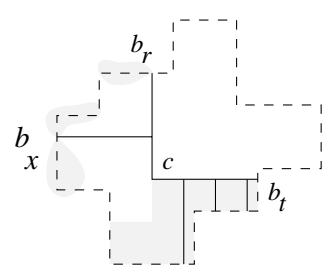

(a)

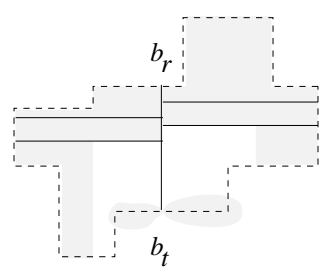

(b)

Figure 3:

The minimal Group 1 tree equals $R C(S)$ minus the longest portion of $R C(S)$ whose interior does not contain any terminal point. This can be determined easily in $O(n)$ time. Group 1 will be dropped from discussion henceforth. Figure 3(a) shows an example of a Group 2 tree and Figure 3(b) shows an example of a Group 3 tree.

\footnotetext{
${ }^{4}$ An interior line or edge is properly incident to another line $\ell$ if the interior line or edge is incident to the interior of $\ell$.
} 
let the two legs of the complete interior corner be incident to exterior vertices $b_{r}$ and $b_{t}$ on $R C(S)$. Let $c$ be the cornervertex. If we duplicate the two legs, then we obtain two subtrees $T$ and $T^{\prime} . T$ is a minimal-length connection of $\left(b_{r}, c\right)$, $\left(b_{t}, c\right)$, and $S\left[b_{r}, b_{t}\right] . T^{\prime}$ is a minimal-length connection of $\left(b_{r}, c\right),\left(b_{t}, c\right)$, and $S\left[b_{t}, b_{r}\right]$. Therefore, if we have computed enough information for us to construct $T$ and $T^{\prime}$, then their union form a candidate for $G_{2}$. By trying all possible complete interior corners, $G_{2}$ can be identified. We analyze the structures of $T$ and $T^{\prime}$ below to introduce common substructures, divisions, gaps, and optimal subtrees. Section 4 describes their definitions.

Common substructures. Consider $T^{\prime}$. If we duplicate the corner-vertex $c$, then $T^{\prime}$ is decomposed into two subtrees $T_{1}^{\prime}$ and $T_{2}^{\prime} . T_{1}^{\prime}$ connects $\left(b_{t}, c\right)$ and $S\left[b_{t}, a_{i}\right]$ and $T_{2}^{\prime}$ connects $\left(b_{r}, c\right)$ and $S\left[a_{i+1}, b_{r}\right]$ for some neighboring points $a_{i}, a_{i+1}$ in $S\left[b_{t}, b_{r}\right]$. By B2, $T_{1}^{\prime}$ and $T_{2}^{\prime}$ enjoys the property that every interior line properly incident to the two legs is a single edge that intersects $R C(S)$. We call $T_{1}^{\prime}$ and $T_{2}^{\prime}$ common substructures. So $T^{\prime}$ is the union of two common substructures and the choice of $a_{i}, a_{i+1}$ must minimize the total length of $T^{\prime}$. This can be done by trying all $O(n) a_{i}, a_{i+1}$ in $S\left[b_{t}, b_{r}\right]$.

Divisions, gaps and optimal subtrees. Consider $T$. W.l.o.g, by B3, we assume that there is exactly one interior edge $f$ properly incident to $\left(b_{r}, c\right)$ that has an endpoint $b_{x}$ on $S\left[b_{r}, b_{t}\right]$. If we duplicate the vertices $b_{r}, b_{x}$ and $c$, then $T$ is decomposed into three parts $T_{1}, T_{2}$ and $T_{3}$. $T_{1}$ is $\left\{f,\left(b_{r}, c\right)\right\}$. $T_{2}$ spans $S\left[b_{r}, b_{x}\right]$ and contains two connected components $T_{21}$ and $T_{22}$, which are optimal Steiner trees for $S\left[b_{r}, a_{i}\right]$ and $S\left[a_{i+1}, b_{t}\right]$ for some neighboring points $a_{i}, a_{i+1}$ in $S\left[b_{r}, b_{x}\right]$. The choice of $a_{i}, a_{i+1}$ must minimize the total length of $T_{2}$ and we call $T_{2}$ a gap. We call $T_{21}$ and $T_{22}$ optimal subtrees. $T_{3}$ spans $S\left[b_{x}, b_{t}\right]$ and contains two connected components $T_{31}$ and $T_{32} . T_{31}$ is an optimal subtree for $S\left[b_{x}, a_{j}\right]$ and $T_{32}$ connects $\left(b_{t}, c\right)$ and $S\left[a_{j+1}, b_{t}\right]$ for some neighboring points $a_{j}, a_{j+1}$ in $S\left[b_{r}, b_{t}\right]$. The choice of $a_{j}, a_{j+1}$ must minimize the total length of $T_{3}$ and we call $T_{3}$ a division. Note that by B2, in $T_{3}$, every interior line properly incident to $\left(b_{t}, c\right)$ is a single edge that intersects $R C(S)$. So $T$ is the minimal-length union of a gap, a division and $\left\{f,\left(b_{r}, c\right)\right\}$ which can be obtained by trying all possible $O(n)$ locations of $b_{x}$. Note that if $f$ does not exist, then we can take $b_{x}=b_{r}$, the gap disappears, and $T$ becomes the union of $\left(b_{r}, c\right)$ and a division.

Thus, $G_{2}$ can be constructed by composing common substructures, gaps, and divisions. From the above discussion, given a blue complete interior corner, it takes $O(n)$ time to construct a candidate for $G_{2}$. There are $O\left(k^{2}\right)$ blue complete interior corners to consider and so $G_{2}$ can be constructed in $O\left(k^{2} n\right)$ time. Consider an example of a Group 3 tree in Figure 3(b). If we duplicate the complete interior line $\left(b_{r}, b_{t}\right)$, then we obtain two subtrees $T$ and $T^{\prime}$. $T$ connects $\left(b_{r}, b_{t}\right)$ and $S\left[b_{r}, b_{t}\right]$ and $T^{\prime}$ connects $\left(b_{t}, b_{r}\right)$ and $S\left[b_{t}, b_{r}\right]$. If we duplicate $b_{t}$ in $T$ and $T^{\prime}$, then both $T$ and $T^{\prime}$ become divisions and they can be put together in $O(1)$ time. However, it is too time-consuming to compute all common substructures, divisions, gaps, and optimal subtrees. Therefore, the construction of $G_{2}$ and $G_{3}$ may sometimes be done indirectly. It still takes $O\left(k^{2} n\right)$ time to construct $G_{2}$ and it will take $O(k n)$ time to construct $G_{3}$. The details are a bit more complicated and are given in appendix A. This summarizes the basic approach to compute $G_{2}$ and $G_{3}$.

The remaining issue is how to compute common substructures, divisions, gaps, and optimal subtrees. We first examine the structure of a division closer. Consider the division $T_{3}$ again in Figure 3(a) which spans $S\left[b_{x}, b_{t}\right]$ and contains $\left(b_{t}, c\right)$. Consider the interior edge properly incident to $\left(b_{t}, c\right)$ and furthest from $b_{t}$. Let its endpoint on $R C(S)$ be $b_{y}$. In $T_{32}$, if we duplicate $b_{y}$, then we obtain an optimal subtree $T_{321}$ which interconnects $S\left[a_{j+1}, b_{y}\right]$ and a common substructure $T_{322}$ which connects $\left(b_{t}, c\right)$ and $S\left[b_{y}, b_{t}\right] . T_{31}$ and $T_{321}$ form a gap for $S\left[b_{x}, b_{y}\right]$. Thus, a division can be defined to be the union of a gap and a common substructure. Section 4 presents the definitions of common substructure, gap, division, and optimal subtree. The algorithms to construct them are described in Sections $5-8$.

\section{Partial solutions}

In the following, let $b_{r}$ and $b_{t}$ be two exterior vertices such that $\left[b_{r}, b_{t}\right]$ lie on three boundary staircases (i.e., $\left[b_{r}, b_{t}\right] \subseteq$ $B_{1} \cup B_{2} \cup B_{3}$, or $B_{2} \cup B_{3} \cup B_{4}$, or $B_{3} \cup B_{4} \cup B_{1}$, or $B_{4} \cup B_{1} \cup B_{2}$.)

Optimal subtrees. Denoted by Tree $\left(b_{r}, b_{t}\right)$. Tree $\left(b_{r}, b_{t}\right) \in \tau\left(S\left[b_{r}, b_{t}\right]\right)$ and either at least one of $b_{r}$ and $b_{t}$ is blue, or $\left(b_{r}, b_{t}\right)$ is a non-blue grid line.

Gap. Denoted by $\operatorname{Gap}\left(b_{r}, b_{t}\right)$. Gap $\left(b_{r}, b_{t}\right)=T_{1} \cup T_{2}$ where $T_{1} \in \tau\left(S\left[b_{r}, a_{i}\right]\right)$ and $T_{2} \in \tau\left(S\left[a_{i+1}, b_{t}\right]\right)$ such that $a_{i}$, $a_{i+1}$ are neighboring points in $S\left[b_{r}, b_{t}\right]$ that minimizes the total length of $G a p\left(b_{r}, b_{t}\right)$. At least one of $b_{r}$ and $b_{t}$ must be blue. 
closed half plane that contains $c$ and bounded by an infinite line through $d$ orthogonal to $\ell .^{5} C\left(b_{r}, b_{t},\left(b_{r}, v\right)\right)$ is defined when $v$ is a grid vertex or exterior vertex, $\left(b_{r}, v\right)$ is a blue interior line and $\left[b_{r}, b_{t}\right] \subseteq$ openspan $\left(\left(b_{r}, v\right)\right) . C\left(b_{r}, b_{t},\left(b_{r}, v\right)\right)$ is the minimal Steiner tree that connects $\left(b_{r}, v\right)$ and $S\left[b_{r}, b_{t}\right]$, and satisfies the following requirement: every interior line incident to $v$ or properly incident to $\left(b_{r}, v\right)$ is a single edge which is orthogonal to $\left(b_{r}, v\right)$ and incident to an exterior vertex in $\left[b_{r}, b_{t}\right]$. We can similarly define a 'clockwise' version $C_{c}\left(b_{r}, b_{t},\left(b_{r}, v\right)\right)$ to connect $\left(b_{r}, v\right)$ and $S\left[b_{t}, b_{r}\right]$ if $\left[b_{t}, b_{r}\right]$ lies on three boundary staircases and $\left[b_{t}, b_{r}\right] \subseteq$ openspan $\left(\left(b_{r}, v\right)\right)$. The same requirements hold for $v$ and $\left(b_{r}, v\right)$, and every interior line incident to $v$ or properly incident to $\left(b_{r}, v\right)$ must be a single edge which is orthogonal to $\left(b_{r}, v\right)$ and incident to an exterior vertex in $\left[b_{t}, b_{r}\right]$.

Division. Denoted by $\operatorname{Div}\left(b_{r}, b_{t},\left(b_{r}, v\right)\right)$. $\operatorname{Div}\left(b_{r}, b_{t},\left(b_{r}, v\right)\right)$ is defined when $b_{t}$ is blue, $v$ is a grid vertex or an exterior vertex, $\left(b_{r}, v\right)$ is a blue interior line, and $\left[b_{r}, b_{t}\right] \subseteq$ openspan $\left(\left(b_{r}, v\right)\right)$ or $b_{t} \notin$ openspan $\left(\left(b_{r}, v\right)\right)$. Div $\left(b_{r}, b_{t},\left(b_{r}, v\right)\right)$ equals $C\left(b_{r}, b_{x},\left(b_{r}, v\right)\right) \cup \operatorname{Gap}\left(b_{x}, b_{t}\right)$ where $b_{x} \in\left[b_{r}, b_{t}\right] \cap$ openspan $\left(\left(b_{r}, v\right)\right)-\left\{b_{t}\right\}$ gives the minimum total length among all exterior vertices in $\left[b_{r}, b_{t}\right] \cap$ openspan $\left(\left(b_{r}, v\right)\right)-\left\{b_{t}\right\}$. We can similarly define a 'clockwise' version $\operatorname{Div}_{c}\left(b_{r}, b_{t},\left(b_{r}, v\right)\right)$ if $\left[b_{t}, b_{r}\right]$ lies on three boundary staircases. The same requirements hold for $b_{r}, v$ and $b_{t}$. Either $\left[b_{t}, b_{r}\right] \subseteq$ openspan $\left(\left(b_{r}, v\right)\right)$ or $b_{t} \notin$ openspan $\left(\left(b_{r}, v\right)\right)$. The formula for $\operatorname{Div}_{c}\left(b_{r}, b_{t},\left(b_{r}, v\right)\right)$ is the same except that $\left[b_{r}, b_{t}\right]$ is replaced by $\left[b_{t}, b_{r}\right]$.

We emphasize again that $\left[b_{r}, b_{t}\right]$ must lie on three boundary staircases in the above definitions. This is required so that all common substructures, divisions, gaps, and optimal subtrees can be computed in $O\left(k^{2} n\right)$ time. Their computation is based on dynamic programming which has $O(k n)$ iterations. In iteration $j$, we compute the above structures for all $\left[b_{r}, b_{t}\right]$ such that $\left|\left[b_{r}, b_{t}\right]\right|=j$ (and subject to the requirements in the definitions), while these structures have been computed for all $\left[b_{r}, b_{t}\right]$ such that $\left|\left[b_{r}, b_{t}\right]\right|<j$ (and subject to the requirements in the definitions). For common substructures $C\left(b_{r}, b_{t},\left(b_{r}, v\right)\right)$ and divisions $\operatorname{Div}\left(b_{r}, b_{t},\left(b_{r}, v\right)\right)$ (and their clockwise versions), we also need to vary $v$. For divisions, there are altogether $O\left(k^{2} n\right)$ things to compute as $b_{r}$ and $b_{t}$ are blue and there are $O(n)$ possible locations for $v$. For common structures, there would be too many $\left(O\left(k n^{2}\right)\right)$ things to compute as $b_{r}$ is blue and there are $O\left(n^{2}\right)$ possible combinations of $b_{t}$ and $v$. So only a subset $(O(k n))$ will be computed explicitly. Other common substructures will be constructed on-line in $O(1)$ time when needed. In each iteration, due to dependency, we must compute gaps first, and then divisions, optimal subtrees, and common substructures in this order.

In dynamic programming, we will build up a 'bigger' partial solution (Steiner tree or forest) by taking the union of a constant number of 'smaller' partial solutions and a constant number of additional tree edges. This union can be implemented in $O(1)$ time by creating a new record for the 'bigger' partial solution which stores pointers to the records for the constituent 'smaller' partial solutions and a constant-size list of the additional tree edges. We denote this union by the standard operator $U$.

\section{$5 \quad$ Gaps}

If both $b_{r}$ and $b_{t}$ are blue, $\operatorname{Gap}\left(b_{r}, b_{t}\right)$ can be computed by returning the minimal Tree $\left(b_{r}, a_{i}\right) \cup \operatorname{Tree}\left(a_{i+1}, b_{t}\right)$ for some neighboring points $a_{i}, a_{i+1} \in S\left[b_{r}, b_{t}\right]$. This takes $O(n)$ time. For the case where either $b_{r}$ or $b_{t}$ is blue and $\operatorname{Gap}\left(b_{r}, b_{t}\right)$ satisfies blue topological properties, we show below that $G a p\left(b_{r}, b_{t}\right)$ can be constructed in $O(1)$ time. Therefore, the total time to compute all gaps is $O\left(k^{2} n\right)$. The additional requirement of blue topological properties is for simplifying the algorithm. If it is not satisfied, then the gap computed may not have the minimal total length. Nevertheless, recall that gaps are used as building blocks for an optimal Steiner tree that satisfies the blue topological properties. Therefore, incorrect gaps cannot be part of the desired optimal Steiner tree. So we are not concerned about constructing them incorrectly. W.l.o.g., assume that $b_{r}$ is blue and so $b_{t}$ is non-blue.

In $G a p\left(b_{r}, b_{t}\right)$, there are three possible topologies: (1) $b_{t}$ has degree zero, (2) $b_{t}$ has degree one and $b_{t}$ is not incident to any interior line, (3) $b_{t}$ is incident to an interior line $\ell$. We compute in $O(1)$ time three forests $F_{1}, F_{2}$ and $F_{3}$ for the three cases and return the minimal one as $\operatorname{Gap}\left(b_{r}, b_{t}\right)$. In case $(1), F_{1}$ is $\operatorname{Gap}\left(b_{r}, a_{j}\right) \cup\left\{b_{t}\right\}$, where $a_{j}$ is the terminal point in $S\left[b_{r}, b_{t}\right]$ closest to $b_{t}$. In case $(2), b_{t}$ has degree one and $G a p\left(b_{r}, b_{t}\right)$ contains $B\left(b_{t-1}, b_{t}\right)$. Thus, $F_{2}$ equals $G a p\left(b_{r}, b_{t-1}\right) \cup B\left(b_{t-1}, b_{t}\right)$. In case $(3)$, since $b_{t}$ is non-blue, $\ell$ is either a complete interior line or the body of a $\mathrm{T}$ (by B5, $\ell$ cannot be a leg of a complete interior corner). We compute in $O(1)$ time two forests $F_{31}$ and $F_{32}$ for these two cases and return the minimal one as $F_{3}$. If $\ell$ is a complete interior line, then $\ell$ does not contain any terminal point by B6 $\left(b_{t}\right.$ is non-blue). Therefore, $F_{31}$ equals the minimal $\operatorname{Gap}\left(b_{r}, b_{z}\right) \cup \operatorname{Tree}\left(b_{z}, b_{t}\right)$, where $b_{z} \in\left[b_{r}, b_{t}\right]$ and $\left(b_{z}, b_{t}\right)$ is a grid line. (There are at most two such $b_{z}$ 's.) Suppose that $\ell$ is the body of a T. Let $b_{x}$ be the blue exterior vertex in $\left[b_{r}, b_{t}\right]$ closest to $b_{r}$. Either $b_{x}$ and $b_{r}$ belong to the same connected component or they belong to

${ }^{5}$ Note that openspan $((c, d)) \neq$ openspan $((d, c))$. 
Thus, $F_{32}$ equals the minimal forest between $B\left(b_{r}, b_{x}\right) \cup \operatorname{Gap}\left(b_{x}, b_{t}\right)$ and $\operatorname{Gap}\left(b_{r}, b_{x}\right) \cup \operatorname{Tree}\left(b_{x}, b_{t}\right)$.

\section{Divisions}

Let $\ell=\left(b_{r}, c\right)$ be a blue complete interior line. Note that it is not necessary that $c \in\left[b_{r}, b_{t}\right]$. Let $v_{0}, v_{1}, v_{2} \cdots$ be the sequence of grid graph vertices on $\ell$ at increasing distances from $b_{r}$ and $v_{0}=b_{r}$. (Note that we do not need to compute the grid graph vertices explicitly. We only need to store the $x$-coordinates ( $y$-coordinates) of all vertical (horizontal) grid lines.) We show how to compute $\operatorname{Div}\left(b_{r}, b_{t}, \ell_{j}\right)$ for all $\ell_{j}=\left(b_{r}, v_{j}\right)$ such that $\left[b_{r}, b_{t}\right] \subseteq$ openspan $\left(\ell_{j}\right)$ or $b_{t} \notin$ openspan $\left(\ell_{j}\right)$ in $O(n)$ time. Thus, the total time to compute all divisions is $O\left(k^{2} n\right)$. The clockwise versions can be computed similarly. The computation is inductive on $j$. Div $\left(b_{r}, b_{t}, \ell_{0}\right)$ equals $\operatorname{Gap}\left(b_{r}, b_{t}\right)$. Consider $j \geq 1$. If $\left[b_{r}, b_{t}\right] \nsubseteq$ openspan $\left(\ell_{j}\right)$ and $b_{t} \in$ openspan $\left(\ell_{j}\right)$, then we can stop the inductive computation. Otherwise, there are two possible topologies in $\operatorname{Div}\left(b_{r}, b_{t}, \ell_{j}\right):(1) v_{j}$ has degree one, (2) $v_{j}$ has degree two. We compute in $O(1)$ time two forests $F_{1}$ and $F_{2}$ for the two cases and return the minimal one as $\operatorname{Div}\left(b_{r}, b_{t}, \ell_{j}\right)$. In case (1), by optimality, removing from $\operatorname{Div}\left(b_{r}, b_{t}, \ell_{j}\right)$ the part of $\ell_{j}$ joining $v_{j-1}$ and $v_{j}$ should produce $\operatorname{Div}\left(b_{r}, b_{t}, \ell_{j-1}\right)$. Thus, $F_{1}$ equals $\operatorname{Div}\left(b_{r}, b_{t}, \ell_{j-1}\right) \cup\left\{\left(v_{j-1}, v_{j}\right)\right\}$. In case $(2), v_{j}$ is incident to an interior edge which intersects $B\left(b_{r}, b_{t}\right)$ at an exterior vertex, say $b_{x}$. So $F_{2}$ equals $C\left(b_{r}, b_{x}, \ell_{j}\right) \cup G a p\left(b_{x}, b_{t}\right)$. To retrieve $b_{x}$, it suffices to retrieve the endpoints of the grid line through $v_{j}$ perpendicular to $\ell$. This can be done by storing the horizontal (vertical) grid lines in an array indexed by their $y$-coordinates ( $x$-coordinates).

\section{Optimal subtrees}

We first define four subsets of $\tau\left(S\left[b_{r}, b_{t}\right]\right)$ (possibly non-disjoint and empty) when $b_{r}$ or $b_{t}$ is blue. W.l.o.g., we assume throughout this section that $\left[b_{r}, b_{t}\right]$ lies on $B_{1} \cup B_{2} \cup B_{3}$. The other cases can be handled similarly. In the following, let $\left\{b_{r}, b_{r+1}, \cdots, b_{t-1}, b_{t}\right\}$ be the sequence $\left[b_{r}, b_{t}\right]$. Let $\beta\left(S\left[b_{r}, b_{t}\right]\right) \subseteq \tau\left(S\left[b_{r}, b_{t}\right]\right)$ be the set of all trees that satisfy the blue topological properties.

Class-1. Let $b_{x}$ be the blue exterior vertex in $\left[b_{r}, b_{t}\right]$ closest to $b_{r}$. Let $b_{y}$ be the blue exterior vertex in $\left[b_{r}, b_{t}\right]$ closest to $b_{t}$. If $b_{r}$ is blue, then the Class- 1 trees are Tree $\left(b_{r}, b_{t-1}\right) \cup B\left(b_{t-1}, b_{t}\right)$ and $B\left(b_{r}, b_{x}\right) \cup T r e e\left(b_{x}, b_{t}\right)$. If $b_{t}$ is blue, then the Class-1 trees are $B\left(b_{r}, b_{r+1}\right) \cup \operatorname{Tree}\left(b_{r+1}, b_{t}\right)$ and Tree $\left(b_{r}, b_{y}\right) \cup B\left(b_{y}, b_{t}\right)$. If both $b_{r}$ and $b_{t}$ are blue, then the Class- 1 trees are the above four trees.

Class-2. A Steiner tree in $\beta\left(S\left[b_{r}, b_{t}\right]\right)$ that contains a blue complete interior line incident to $b_{r}$ or $b_{t}$.

Class-3. A Steiner tree in $\beta\left(S\left[b_{r}, b_{t}\right]\right)$. If there exists $b_{x} \in\left[b_{r}, b_{t}\right]-\left\{b_{r}, b_{t}\right\}$ such that $\left(b_{r}, b_{x}\right)$ or $\left(b_{t}, b_{x}\right)$ is a grid line, then Tree $\left(b_{r}, b_{x}\right) \cup \operatorname{Tree}\left(b_{x}, b_{t}\right)$ is a Class- 3 tree. Note that there are at most two such choices for $b_{x}$ for any $\left[b_{r}, b_{t}\right]$.

Class-4. A Steiner tree in $\beta\left(S\left[b_{r}, b_{t}\right]\right)$ that contains a blue complete interior corner such that one of its legs is incident to $b_{r}$ or $b_{t}$.

Theorem 7.1 Let $E($ Class- 1$) \subseteq \tau\left(S\left[b_{r}, b_{t}\right]\right)$ be the set of all trees that can be transformed into a Class- 1 tree by sliding edge(s) and/or flipping corner(s). If $b_{r}$ or $b_{t}$ is blue and Tree $\left(b_{r}, b_{t}\right) \in \beta\left(S\left[b_{r}, b_{t}\right]\right)-E($ Class- 1$)$, then Tree $\left(b_{r}, b_{t}\right) \in$ $\bigcup_{j=2}^{4}$ Class $-j$.

Proof W.l.o.g., assume that $b_{r}$ is blue ( $b_{t}$ may or may not be blue). Suppose that Tree $\left(b_{r}, b_{t}\right) \in \beta\left(S\left[b_{r}, b_{t}\right]\right)-$ $E$ (Class-1). Tree $\left(b_{r}, b_{t}\right)$ must contain some interior line $\ell$ incident to $b_{t}$. Otherwise, Tree $\left(b_{r}, b_{t}\right)$ equals $\operatorname{Tree}\left(b_{r}, b_{t-1}\right) \cup$ $B\left(b_{t-1}, b_{t}\right)$ which is in Class-1, a contradiction. By blue topological properties, $\ell$ is a complete interior line, a leg of a complete interior corner, or the body of a $\mathrm{T}$. If $\ell$ is a complete interior line, then Tree $\left(b_{r}, b_{t}\right)$ is in Class- 2 or Class- 3 . If $\ell$ is a leg of a complete interior corner, then Tree $\left(b_{r}, b_{t}\right)$ is in Class-4. If $\ell$ is the body of an interior $\mathrm{T}$, then $\ell$ must be horizontal due to the fact that $\left[b_{r}, b_{t}\right]$ lie on $B_{1} \cup B_{2} \cup B_{3}$ and $\operatorname{Tree}\left(b_{r}, b_{t}\right)$ lies inside $R C\left(S\left[b_{r}, b_{t}\right]\right)$. We claim that the upper endpoint of the T-head is an exterior vertex $b_{p}$ on the boundary as shown in Figure 4(a). By $\mathrm{B} 5$ or B6, $b_{p}$ is blue. Moreover, $b_{p}=b_{r}$. (Otherwise, Tree $\left(b_{r}, b_{t}\right)$ equals $B\left(b_{r}, b_{p}\right) \cup \operatorname{Tree}\left(b_{p}, b_{t}\right)$ which is in Class-1, a contradiction). This implies that Tree $\left(b_{r}, b_{t}\right)$ is in Class-2 or Class-4. If the upper endpoint of the T-head is not on the boundary, then it is an interior corner-vertex. There are two possible configurations as shown in Figures 4(b) and (c), depending on whether there is any interior edge properly incident to the horizontal leg. We derive below a contradiction in both cases. By B5, $b_{q}$ in Figures $4(\mathrm{~b})$ and (c) is blue. Also, $b_{q} \neq b_{r}$ as Tree $\left(b_{r}, b_{t}\right)$ would lie outside $R C\left(S\left[b_{r}, b_{t}\right]\right)$ otherwise. We flip the complete interior corner (in Figure $4(c), b_{q}$ must lie on $B_{3}$; otherwise, this flipping would increase overlapping with the boundary which violates B4). After the flipping, we obtain the tree $B\left(b_{r}, b_{q}\right) \cup \operatorname{Tree}\left(b_{q}, b_{t}\right)$ which is in Class-1. This contradicts that Tree $\left(b_{r}, b_{t}\right) \notin E($ Class- 1$)$. 


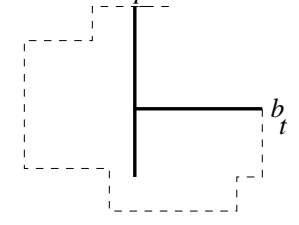

(a)

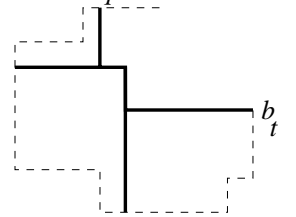

(b)

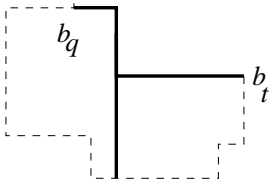

(c)

Figure 4:

Theorem 7.1 means that if $b_{r}$ or $b_{t}$ is blue, then it suffices to construct a Steiner tree $C T_{j}$ whose total length is not greater than that of the minimal Steiner tree in Class- $j$, for $1 \leq j \leq 4$. The minimal tree among $C T_{j}, 1 \leq j \leq 4$, is returned as Tree $\left(b_{r}, b_{t}\right)$. We do not construct the minimal Steiner tree in Class- $j, 1 \leq j \leq 4$, directly because we will slide edge(s) to transform them so that we can use 'smaller' partial solutions. If $\beta\left(S\left[b_{r}, b_{t}\right]\right)-E$ (Class- 1$)$ is empty, then the Tree $\left(b_{r}, b_{t}\right)$ constructed may not have the minimal total length. Nevertheless, recall that optimal subtrees are used as building blocks for an optimal Steiner tree that satisfies the blue topological properties. Therefore, incorrect optimal subtrees cannot be part of the desired optimal Steiner tree. So we are not concerned about constructing them incorrectly. By similar argument, we also make the following remark:

Remark. When $\left(b_{r}, b_{t}\right)$ is a non-blue grid line, it suffices to construct a Tree $\left(b_{r}, b_{t}\right)$ that also belongs to $\beta\left(S\left[b_{r}, b_{t}\right]\right)$. Sections $7.1-7.4$ describe how to compute $C T_{j}, 1 \leq j \leq 4$, in $O(k)$ time. Section 7.5 describes how to compute Tree $\left(b_{r}, b_{t}\right)$ in $O(1)$ time when $\left(b_{r}, b_{t}\right)$ is a non-blue grid line. Thus, the total time to compute all optimal subtrees is $O\left(k^{2} n\right)$.

\subsection{Class-1}

We compute at most four candidate trees as described in the definition of Class- 1 and pick the minimal one as $C T_{1}$. To do this in $O(1)$ time, at each exterior vertex, we need to keep pointers to the neighboring exterior vertices and neighboring blue exterior vertices. This can be done in preprocessing by an $O(n)$-time scan of all exterior vertices in cyclic order around $R C(S)$.

\section{$7.2 \quad$ Class-2}

For each blue grid line $\left(b_{r}, b_{x}\right)$ or $\left(b_{t}, b_{x}\right)$, where $b_{x} \in\left[b_{r}, b_{t}\right]$, we compute a candidate tree in $O(1)$ time. There are at most two such blue grid lines for any $\left[b_{r}, b_{t}\right]$. The minimal candidate tree is returned as $C T_{2}$. Suppose that the minimal Class-2 tree contains $\left(b_{r}, b_{x}\right)$. (The case for $\left(b_{t}, b_{x}\right)$ is symmetric.) If $b_{t} \in \operatorname{openspan}\left(\left(b_{x}, b_{r}\right)\right)$, the corresponding candidate tree equals $\operatorname{Div}\left(b_{r}, b_{x},\left(b_{r}, b_{x}\right)\right) \cup C\left(b_{x}, b_{t},\left(b_{x}, b_{r}\right)\right)$. The union takes $O(1)$ time. This processing demands that for any exterior vertex $c$, we keep pointer(s) to the other endpoint(s) of the vertical/horizontal grid line(s) incident to $c$. Since there are $O(n)$ grid lines, this can be done in $O(n)$ time in preprocessing. When $b_{t} \notin$ openspan $\left(\left(b_{x}, b_{r}\right)\right), b_{r}$ must be on $B_{3},\left(b_{s}, b_{r}\right)$ must be vertical, and $b_{t}$ must be on $B_{1}$ and above $b_{r}$. Thus, $R C\left(S\left[b_{r}, b_{t}\right]\right)=R C\left(S\left[b_{r}, b_{t-1}\right]\right) \cup B\left(b_{t-1}, b_{t}\right)$ and so Tree $\left(b_{r}, b_{t}\right)$ equals Tree $\left(b_{r}, b_{t-1}\right) \cup B\left(b_{t-1}, b_{t}\right)$ which is in Class-1. Class-1 has been handled before and so we can ignore the case $b_{t} \notin$ openspan $\left(\left(b_{x}, b_{r}\right)\right)$.

\subsection{Class-3}

$C T_{3}$ is the minimal Tree $\left(b_{r}, b_{x}\right) \cup \operatorname{Tree}\left(b_{x}, b_{t}\right)$, where $b_{x} \in\left[b_{r}, b_{t}\right]$ and $\left(b_{r}, b_{x}\right)$ or $\left(b_{t}, b_{x}\right)$ is a grid line (not necessarily blue). There are at most two such grid lines for any $\left[b_{r}, b_{t}\right]$. Note that if $b_{r}$ is blue, then $b_{x}$ is also blue and so both Tree $\left(b_{r}, b_{x}\right)$ and Tree $\left(b_{x}, b_{t}\right)$ are available. If $b_{r}$ is non-blue, then $\left(b_{r}, b_{x}\right)$ is a non-blue grid line and $b_{t}$ must be blue by definition. So Tree $\left(b_{r}, b_{x}\right)$ and Tree $\left(b_{x}, b_{t}\right)$ are also available. (Recall that Section 7.5 describes the computation of Tree $\left(b_{r}, b_{t}\right)$ when $\left(b_{r}, b_{t}\right)$ is a non-blue grid line.)

\subsection{Class -4}

There are four possible configurations depending on the orientation of the blue complete interior corner. See Figure 5. We compute four candidate trees in $O(k)$ time which are in the four configurations shown. The minimal tree is returned as $C T_{4}$. Configurations $\mathrm{A}$ and $\mathrm{B}$ are symmetric and configurations $\mathrm{C}$ and $\mathrm{D}$ are symmetric. Therefore, we only discuss how to compute the candidate trees for configurations $\mathrm{A}$ and $\mathrm{C}$. 


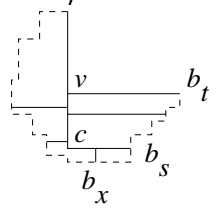

(a)

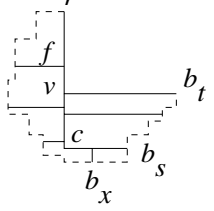

(b)

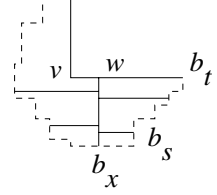

(c)

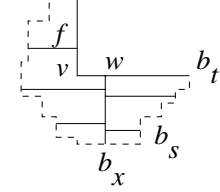

(d)

Figure 7:

(2) $b_{s} \neq b_{t}$. We compute in $O(k)$ time two trees $T_{1}$ and $T_{2}$ for the two cases and return the minimal one as the desired tree of configuration $\mathrm{C}$.

Consider $T_{1}$. By B3, one leg of $\left(b_{r}, c, b_{t}\right)$ has at most one interior edge properly incident to it. There are four different cases depending on $\left(b_{r}, c\right)$ or $\left(b_{t}, c\right)$ has zero or one interior edge properly incident to it. We compute a candidate tree for each case and then return the minimal tree as $T_{1}$. For the case where no interior edge is properly incident to $\left(b_{t}, c\right)$, the candidate tree equals $\operatorname{Div}\left(b_{r}, b_{t},\left(b_{r}, c\right)\right) \cup\left\{\left(b_{t}, c\right)\right\}$. Consider the case where exactly one interior edge $f$ is properly incident to $\left(b_{t}, c\right)$. Let $b_{x}$ be the endpoint of $f$ on the boundary which is blue by B5. If $b_{x}$ is known, then the candidate tree equals $\operatorname{Div}\left(b_{r}, b_{x},\left(b_{r}, c\right)\right) \cup G a p\left(b_{x}, b_{t}\right) \cup\left\{\left(b_{t}, c\right), f\right\}$. We try all $O(k)$ positions for $b_{x}$ in $\left[b_{r}, b_{t}\right]$ to find the position that minimizes the total length. This takes $O(k)$ time. The two cases where at most one interior edge is properly incident to $\left(b_{r}, c\right)$ is handled similarly.

Consider $T_{2}$. First, by a proof similar to that of Lemma 7.1, we can assume that $b_{t}$ is the endpoint of an interior edge properly incident to $\left(b_{r}, c\right)$. By $\mathrm{B} 1$, interior edges properly incident to $\left(b_{r}, c\right)$ are alternating. By $\mathrm{B} 2$, B3 and $\mathrm{B} 5$, there is at most one interior edge properly incident to $\left(b_{t}, c\right)$ and this interior edge (if exists) is incident to a blue node $b_{x}$. If no interior edge is properly incident to $\left(b_{t}, c\right)$, then we take $b_{x}$ to be $b_{s}$. There are two cases: (1) same number of interior edges are properly incident to both sides of $\left(b_{r}, c\right)$ (Figure $7(\mathrm{a})$ ), $(2)$ there is one more interior edge properly incident to the left side of $\left(b_{r}, c\right)$ than the right side (Figure $7(\mathrm{~b})$ ). We compute in $O(k)$ time two trees $T_{21}$ and $T_{22}$ and return the minimal one as $T_{2}$. In case (1), we convert Figure $7(\mathrm{a})$ to Figure $7(\mathrm{c})$. $T_{21}$, which is the tree in Figure $7(c)$, equals $\operatorname{Div}_{c}\left(b_{x}, b_{r},\left(b_{x}, w\right)\right) \cup C\left(b_{x}, b_{t},\left(b_{x}, b_{t}\right)\right) \cup\left\{\left(b_{r}, v\right),(v, w)\right\}$, which can be constructed in $O(1)$ time if the position of $b_{x}$ is known. We try all $O(k)$ positions for $b_{x}$ in $\left[b_{r}, b_{t}\right]$ to find the position that minimizes the total length. In case (2), there is one interior edge, say $f$, properly incident to $\left(b_{r}, v\right)$. Let $b_{z}$ be the exterior vertex in $\left[b_{r}, b_{t}\right]$ such that $\left(b_{z}, b_{t}\right)$ is a horizontal grid line. Let $b_{p}$ be last node in the sequence $\left[b_{r}, b_{z}\right]$ (recall that a node is a terminal point or an inner boundary corner vertex). Let $b_{q}$ be the lower endpoint of the vertical boundary edge that contains $b_{z}$. Let $a_{p}\left(a_{q}\right)$ be the terminal point closest to $b_{p}\left(b_{q}\right)$ in $S\left[b_{p}, b_{x}\right]\left(S\left[b_{q}, b_{x}\right]\right)$. Let $g$ be the interior edge immediately below $f$ and properly incident to $(v, c)$. The following lemma can be proved (the proof is given in appendix $\mathrm{B}$ ).

Lemma 7.2 Let $T$ be a tree of configuration $C$ such that $T$ cannot be converted to a Class- 1 tree or a tree of configuration $A$ by sliding edges and flipping corners. If the total length of $T$ equals that of Tree $\left(b_{r}, b_{t}\right)$, then one of the following holds:

1. $f$ can be slid downward until it contains $b_{p}$, and after the sliding, $b_{p}\left(a_{p}\right)$ is connected by a tree path in $T$ to $\left(b_{r}, v\right)((v, c))$ that uses $f(g)$,

2. $b_{q}$ is a terminal point, $f$ can be slid downward until it contains $b_{z}$, and $b_{q}\left(a_{q}\right)$ is connected by a tree path in $T$ to $\left(b_{r}, v\right)((v, c))$ that uses $f(g)$.

We convert Figure $7(\mathrm{~b})$ to Figure $7(\mathrm{~d})$ by sliding edge(s). $T_{21}$ to be computed is the tree in Figure $7(\mathrm{~d})$. We assume that the total of the tree in Figure $7(\mathrm{~d})$ equals to that of $\operatorname{Tree}\left(b_{r}, b_{t}\right)$. Otherwise, there is no point in computing $T_{21}$. By definition and Lemma $7.2, a_{p}$ and $a_{q}$ are below $v$ and so $a_{p}, a_{q} \in$ openspan $\left(\left(b_{x}, w\right)\right)$. Lemma 7.2 implies that $S\left[a_{p}, b_{x}\right]$ (or $S\left[a_{q}, b_{x}\right]$ ) is connected together with $\left(b_{x}, w\right)$ to form a common substructure $C_{c}\left(b_{x}, a_{p},\left(b_{x}, w\right)\right.$ ) $\left(C_{c}\left(b_{x}, a_{q},\left(b_{x}, w\right)\right)\right)$. Given $b_{t}$, we can access $b_{z}$ and hence $b_{p}$ in $O(1)$ time. By an $O(n)$-time preprocessing, we can keep pointers at each exterior vertex $c$ to the two endpoints of the boundary edge containing $c$. Thus, $b_{q}$ can also be accessed in $O(1)$ time. We compute in $O(k)$ time two trees $T_{221}$ and $T_{222}$ for the two cases stated in Lemma 7.2 and return the minimal one as $T_{22}$. In the first case, we slide $f$ downward until it is incident to $b_{p}$. $T_{221}$, which is the resulting tree, equals $G a p\left(b_{r}, b_{p}\right) \cup C_{c}\left(b_{x}, a_{q},\left(b_{x}, w\right)\right) \cup C\left(b_{x}, b_{t},\left(b_{x}, w\right)\right) \cup\left\{f,\left(b_{r}, v\right),(v, w)\right\}$ provided that the position of $b_{x}$ is known. In the second case, since $b_{z}$ and $b_{q}$ are contained by the same boundary edge, the tree path connecting $b_{q}$ to $b_{z}$ lies on $B\left(b_{z}, b_{q}\right)$. We slide $f$ downward until it is incident to $b_{z}$. $T_{222}$, which is the resulting tree, equals $G a p\left(b_{r}, b_{z}\right) \cup B\left(b_{z}, b_{q}\right) \cup C_{c}\left(b_{x}, a_{q},\left(b_{x}, w\right)\right) \cup C\left(b_{x}, b_{t},\left(b_{x}, w\right)\right) \cup\left\{f,\left(b_{r}, v\right),(v, w)\right\}$ provided that the position of $b_{x}$ is known. We try all $O(k)$ positions for $b_{x}$ to minimize the total length of $T_{221}$ and $T_{222}$. 
Recall the remark in the beginning of Section 7 that Tree $\left(b_{r}, b_{t}\right)$ can be assumed to satisfy the blue topological properties. Since $\left(b_{r}, b_{t}\right)$ is a non-blue grid line, $b_{r}$ and $b_{t}$ lie in the interior of two opposite boundary edges. This implies that $\left(b_{r+1}, b_{t-1}\right)$ is also a (possibly blue) grid line and Tree $\left(b_{r+1}, b_{t-1}\right)$ is available. In Tree $\left(b_{r}, b_{t}\right)$, there are two possible topologies: (1) no interior edge is incident to $b_{r}$ or $b_{t},(2)$ an interior line $\ell$ is incident to $b_{r}$ or $b_{t}$. We compute in $O(1)$ time two trees $T_{1}$ and $T_{2}$ for the two cases and return the minimal one as $T r e e\left(b_{r}, b_{t}\right)$. In case (1), $T_{1}$ clearly equals $B\left(b_{r}, b_{r+1}\right) \cup T r e e\left(b_{r+1}, b_{t-1}\right) \cup B\left(b_{t-1}, b_{t}\right)$. In case $(2), \ell$ cannot be a T-body; otherwise, the T-head must lie partially outside $R C\left(S\left[b_{r}, b_{t}\right]\right)$. By $\mathrm{B} 5$, since $\ell$ is non-blue, $\ell$ cannot be a leg of a complete interior corner. If $\ell$ is a (non-blue) complete interior line, then $\ell=\left(b_{r}, b_{t}\right)$ and $\ell$ does not contain any Steiner point by B6. Let $a_{q}$ be the terminal point in $S\left[b_{r}, b_{t}\right]$ closest to $b_{t}$. Let $\left(b_{s}, a_{q}\right)$ be a grid line parallel to $\ell$. Let $b_{p}$ be the blue exterior vertex in $\left[b_{r}, b_{t}\right]$ closest to $b_{r}$. $b_{r}$ must be connected by a tree path to $b_{p}$ or $b_{s}$, whichever is closer to $b_{r}$ in $\left[b_{r}, b_{t}\right]$, such that the tree path lies on the boundary edge containing $b_{r}$. Thus, if $b_{p}\left(b_{s}\right)$ is closer to $b_{r}$, then $T_{2}$ equals $B\left(b_{r}, b_{p}\right) \cup \operatorname{Tree}\left(b_{p}, a_{q}\right) \cup\{\ell\}\left(B\left(b_{r}, b_{s}\right) \cup \operatorname{Tree}\left(b_{s}, a_{q}\right) \cup\{\ell\}\right)$.

\section{Common substructures}

Let $\ell=\left(b_{r}, v\right)$ be a blue complete interior line such that $\left[b_{r}, b_{t}\right] \subseteq$ openspan $(\ell)$. There are at most two such complete interior lines incident to $b_{r}$ and we compute $C\left(b_{r}, b_{t}, \ell\right)$ in $O(1)$ time for each such $\ell$. For any grid vertex $w$ on $\ell$ such that $\left[b_{r}, b_{t}\right] \subseteq$ openspan $\left(\left(b_{r}, w\right)\right)$, whenever $C\left(b_{r}, b_{t},\left(b_{r}, w\right)\right)$ is needed, it can be constructed on-line in $O(1)$ time as $\left(C\left(b_{r}, b_{t}, \ell\right)-\{\ell\}\right) \cup\left\{\left(b_{r}, w\right)\right\}$. The correctness follows from the fact all interior edges incident to $\ell$ in $C\left(b_{r}, b_{t}, \ell\right)$ are also incident to $\left(b_{r}, w\right)$. We describe below how to compute $C\left(b_{r}, b_{t}, \ell\right)$ in $O(1)$ time. The clockwise versions can be handled similarly. Since there $O(k n)$ such common substructures, the total processing time is thus $O(k n)$. Let $\left[b_{r}, b_{t}\right]$ be $\left\{b_{r}, b_{r+1}, \cdots, b_{t-1}, b_{t}\right\}$. W.l.o.g., we assume that $\ell$ is vertical and $b_{r}$ is the upper endpoint of $\ell$. See Figure 8 . There

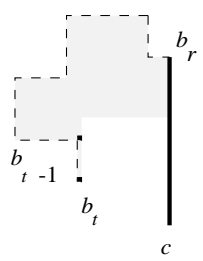

(a)

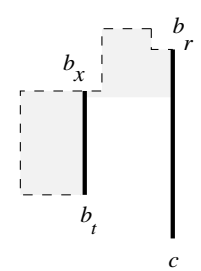

(b)

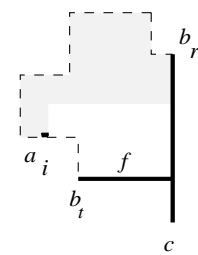

(c)

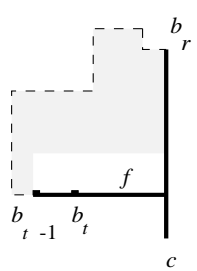

(d)

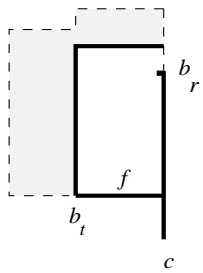

(e)

Figure 8:

are two possible topologies in $C\left(b_{r}, b_{t}, \ell\right)$ : (1) no interior edge is incident to $b_{t}$ and $\ell,(2)$ an interior edge $f$ is incident to $b_{t}$ and $\ell$. We compute in $O(1)$ time two trees $T_{1}$ and $T_{2}$ for the two cases and return the minimal one as $C\left(b_{r}, b_{t}, \ell\right)$.

Case (1): There are two possible topologies : (1.1) no interior edge is properly incident to $\ell,(1.2)$ some interior edge is properly incident to $\ell$. We compute two trees $T_{11}$ and $T_{12}$ for the two cases and return the minimal one as $T_{1}$. In case (1.1), $T_{11}$ equals $T r e e\left(b_{r}, b_{t}\right) \cup\{\ell\}$. In case (1.2), traverse a tree path $P$ from $b_{t}$ to $b_{r}$ until we reach an exterior vertex. We flip all left turns on $P$ and so $P$ is either $B\left(b_{t-1}, b_{t}\right)$ or a complete interior line $\left(b_{x}, b_{t}\right)$. See Figures $8(\mathrm{a})$ and (b). ( $P$ cannot contain a right bend; otherwise, there cannot be any interior edge properly incident to $\ell$ as $P$ has to terminate at an exterior vertex.) Thus, $T_{12}$ equals the minimal tree between $C\left(b_{r}, b_{t-1}, \ell\right) \cup B\left(b_{t-1}, b_{t}\right)$ and $C\left(b_{r}, b_{x}, \ell\right) \cup \operatorname{Tree}\left(b_{x}, b_{t}\right)$. (There is at most one $b_{x} \in\left[b_{r}, b_{t}\right]$ such that $\left(b_{x}, b_{t}\right)$ is a grid line.)

Case (2): There are three possible topologies: (2.1) $b_{t}$ has degree one, (2.2) $b_{t}$ has degree more than one and $b_{t}$ is not incident to a tree edge perpendicular to $f,(2.3) b_{t}$ is incident to a tree edge $g$ perpendicular to $f$. We compute two trees $T_{21}$ and $T_{22}$ for the first two cases and return the minimal one as $T_{2}$. We argue that case (2.3) has been handled before. In case (2.1) (Figure 8(c)), $T_{21}$ equals $C\left(b_{r}, a_{i}, \ell\right) \cup\{f\}$, where $a_{i}$ is the terminal point in $S\left[b_{r}, b_{t}\right]$ closest to $b_{t}$. In case $(2.2)$, by convexity, the extra tree edge incident to $b_{t}$ must contain $B\left(b_{t-1}, b_{t}\right)$ and it is aligned with $f$. See Figure $8(\mathrm{~d})$. Thus, $T_{22}$ equals the tree $T_{2}$ constructed when computing $C\left(b_{r}, b_{t-1}, \ell\right)$. In case $(2.3)$, traverse a tree path $P$ from $b_{t}$ starting along $g$ and making a right turn whenever possible, until an exterior vertex is reached. Similar to case (1.2) above, after flipping all left turns on $P$, either $P$ is $B\left(b_{t-1}, b_{t}\right)$ or $P$ is a complete interior line or $P$ contains a right bend as shown in Figure 8(e). In all three cases, $f$ can be slid upward and this reduces case (2.3) to some case considered in case (1). 
We present $O\left(k^{2} n\right)$-time algorithms for computing new structures (common substructures, division, gaps, and optimal subtrees) of a MRST for an extremal point set. Combined with the proof in Appendix A, we obtain an $O\left(k^{2} n\right)$-time algorithm to compute an optimal Steiner tree for an extremal point set. This strictly improves upon previous results in $[12,4]$. When the terminal points are on the boundary of a rectilinear polygon, it is interesting to see if similar techniques can be used to obtain a fast algorithm.

\section{References}

[1] P. Agarwal and M. Shing, Algorithms for the Specific Cases of Rectilinear Steiner Trees: I. Points on the Boundary of a Rectilinear Rectangle, Networks, 20 (1990), pp. 453-485.

[2] A. Aho, M. Garey, and F. Hwang, Rectilinear Steiner Trees: Efficient Special-Case Algorithms, Networks, 7 (1977), pp. 37-58.

[3] M. Bern, Faster Exact Algorithms for Steiner Tree in Planar Networks, Networks, 7 (1990), pp. 109-120.

[4] S.W. Cheng, A. Lim, and C. Wu, Optimal Rectilinear Steiner Tree for Extremal Point Sets, in Proceedings of International Symposium on Algorithms and Computation (ISAAC), 1993, LNCS 762, pp. 523-532.

[5] J. Cohoon, D. Richards, and J. Salowe, An Optimal Steiner Tree Algorithm for a Net Whose Terminals Lie on the Perimeter of a Rectangle, IEEE Transactions on Computer-Aided Design, 9 (1990), pp. 398-407.

[6] R. E. Erickson, C. Monma, and A. F. Veinott, Send-and-split Method for Minimum-cost Network Flows, Mathematical Operation Research, 12 (1987), pp. 634-664.

[7] M. R. Garey and D. S. Johnson, Computers and Intractability, Freeman, San Francisco, 1979.

[8] F. K. Hwang and D. S. Richards, Steiner Tree Problems, Networks, 22 (1992), pp. 55-89.

[9] M. Kaufmann, S. Gao, and K. Thulasiraman, On Steiner Minimal Trees in Grid Graphs and Its Application to VLSI Routing, in Proceedings of International Symposium on Algorithms and Computation (ISAAC), 1994, LNCS 834, pp. 351-359.

[10] C.E. Leiserson and F.M. Maley, Algorithms for Routing and Testing Routability of Planar VLSI Layouts, Proc. 7th Ann. Symp. Theory of Comput., 1985, pp. 69-78.

[11] J. Provan, Convexity and the Steiner Tree Problem, Networks, 18 (1988), pp. 55-72.

[12] D. Richards and J. Salowe, A Linear-Time Algorithm to Construct a Rectilinear Steiner Minimal Tree for $k$ Extremal Point Sets, Algorithmica, 7 (1992), pp. 247-276.

[13] J.D. Ullamn, Computatinal Aspects of VLSI, Computer Science Press, 1984.

[14] Y. Y. Yang and O. Wing. Optimal and Suboptimal Solution Algorithms for the Wiring Problem. Proc. IEEE International Symposium on Circuit Theory, pages 154-158, 1972. 
We first repeat the definitions of Group- $j$ and $G_{j}, 1 \leq j \leq 3$, in Section 3. We define three groups of Steiner trees for $S$ that satisfy the blue topological properties. Group 1 contains no interior edge. Group 2 contains some complete interior corners. Group 3 contains some complete interior line but no complete interior corner. For $1 \leq j \leq 3$, the tree $G_{j}$ to be computed is a Steiner tree with total length no greater than the minimal tree in Group- $j$.

We shall show that $G_{j}, 1 \leq j \leq 3$, can be computed in $O\left(k^{2} n\right)$ time. Then the minimal tree among $G_{1}, G_{2}$, and $G_{3}$ is returned as the desired optimal Steiner tree for the input extremal point set. To this end, we first compute the structures defined in Section 4: common substructures $C\left(b_{r}, b_{t},\left(b_{r}, v\right)\right.$ ) (and the clockwise version), divisions $\operatorname{Div}\left(b_{r}, b_{t},\left(b_{r}, v\right)\right.$ ) (and the clockwise version), gaps $G a p\left(b_{r}, b_{t}\right)$, and optimal subtrees Tree $\left(b_{r}, b_{t}\right)$. The readers are referred to Section 4 for their definitions. We just point out here that $\left[b_{r}, b_{t}\right]$ are required to lie on three boundary staircases ${ }^{6}$. We call this collection of structures the complete set of partial solutions and we assume that they have been computed using the algorithms in Section $5-8$.

We also need to compute divisions for some more sequences $\left[b_{r}, b_{t}\right]$. Recall that for $\left[b_{r}, b_{t}\right]$ lying on three boundary staircases, $\operatorname{Div}\left(b_{r}, b_{t},\left(b_{r}, v\right)\right)$ equals the minimal $C\left(b_{r}, b_{x},\left(b_{r}, v\right)\right) \cup G a p\left(b_{x}, b_{t}\right)$ for some $b_{x} \in$ openspan $\left(\left(b_{r}, v\right)\right) \cap\left[b_{r}, b_{t}\right]-$ $\left\{b_{t}\right\}$. Define $\operatorname{span}\left(\left(b_{r}, v\right)\right)$ to be the region between two infinite lines through $b_{r}$ and $v$ and perpendicular to $\left(b_{r}, v\right)$. Now, we also compute $\operatorname{Div}\left(b_{r}, b_{t},\left(b_{r}, v\right)\right)$ for $\left[b_{r}, b_{t}\right]$ such that that: (1) $\left[b_{r}, b_{t}\right]$ contains exterior vertices from all four boundary staircases, and (2) $b_{r}$ and $b_{t}$ do not lie on the same boundary staircase. In such cases, $\operatorname{Div}\left(b_{r}, b_{t},\left(b_{r}, v\right)\right)$ is defined to be the minimal $C\left(b_{r}, b_{x},\left(b_{r}, v\right)\right) \cup G a p\left(b_{x}, b_{t}\right)$ for some $b_{x} \in \operatorname{span}\left(\left(b_{r}, v\right)\right) \cap\left[b_{r}, b_{t}\right]$. Similarly, we can define a clockwise version $\operatorname{Div}_{c}\left(b_{r}, b_{t},\left(b_{r}, v\right)\right)$ to be the minimal $C_{c}\left(b_{r}, b_{x},\left(b_{r}, v\right)\right) \cup G a p\left(b_{t}, b_{x}\right)$ for some $b_{x} \in \operatorname{span}\left(\left(b_{r}, v\right)\right) \cap\left[b_{t}, b_{x}\right]$. We call such an additional division an extra-division and we call the collection of all extra-divisions the complete set of extra-divisions. Since $b_{r}$ and $b_{t}$ are required not to lie on the same boundary staircase, for all $b_{x} \in \operatorname{span}\left(\left(b_{r}, v\right)\right)$, $C\left(b_{r}, b_{x},\left(b_{r}, v\right)\right)$ and $G a p\left(b_{x}, b_{t}\right)$ are available as $\left[b_{r}, b_{x}\right]$ and $\left[b_{x}, b_{t}\right]$ must lie on three boundary staircases. Thus, extra-divisions can be computed by composing gaps and common substructures which are available. Moreover, since the formula for extra-divisions are very similar to that for divisions, extra-divisions for all appropriate $\left[b_{r}, b_{t}\right]$ can be computed in $O\left(k^{2} n\right)$ time by a method similar to the one described in Section 6 for divisions.

Theorem A.1 Given an extremal point set $S$ of size $n$, the optimal Steiner tree for $S$ can be computed in $O\left(k^{2} n\right)$ time if the complete set of partial solutions and the complete set of extra-divisions are available.

Proof $G_{1}$ is $R C(S)$ minus the longest portion of the boundary between two adjacent terminal points on the boundary. This can be found easily in $O(n)$ time. It suffices to compute $G_{2}$ and $G_{3}$ such that they satisfy the blue topological properties in Section 3.

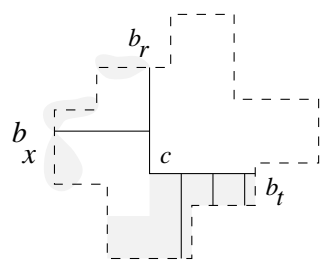

(a)

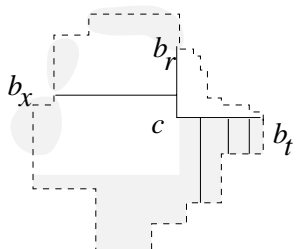

(b)

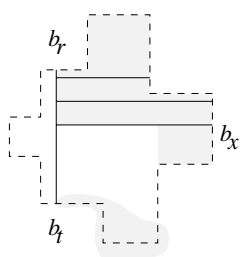

(c)

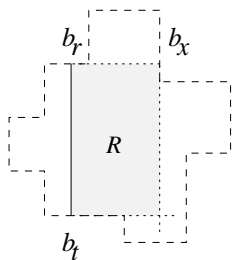

(d)

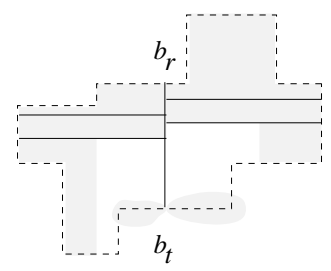

(e)

Figure 9: $G_{2}$ and $G_{3}$.

The minimal Group-2 tree contains a complete interior corner whose legs are incident to two blue exterior vertices $b_{r}$ and $b_{t}$. Let $c$ be the corner-vertex. By B3, there are $O\left(k^{2}\right)$ possible choices for $b_{r}$ and $b_{t}$. For each pair of $b_{r}$ and $b_{t}$, there are three possible cases as listed below. We describe below how to compute in $O(n)$ time a tree for each case. The minimal tree among all the $O\left(k^{2}\right)$ cases is the desired $G_{2}$.

Case (1.1): $b_{r}$ and $b_{t}$ lie on opposite boundary staircases (i.e., $B_{1}$ and $B_{3}$ as in Figure $9(a)$ or $B_{2}$ and $B_{4}$ ). The subtree that connects $S\left[b_{t}, b_{r}\right]$ and the complete interior corner equals $C\left(b_{t}, a_{i},\left(b_{t}, c\right)\right) \cup C_{c}\left(b_{r}, a_{i+1},\left(b_{r}, c\right)\right)$ for some neighboring points $a_{i}, a_{i+1} \in S\left[b_{t}, b_{r}\right]$. The choice of $a_{i}, a_{i+1} \in S\left[b_{t}, b_{r}\right]$ should minimize the total length and this can be found in $O(n)$ time. It remains to describe how to compute the subtree connecting $S\left[b_{r}, b_{t}\right]$ and the complete interior corner. Denote this subtree by $T$. By B3, there are four possible cases depending on $\left(b_{r}, c\right)$ or $\left(b_{t}, c\right)$ has zero or one interior edge properly incident to it. We compute four candidate trees for the four cases and return the

\footnotetext{
${ }^{6} B_{1} \cup B_{2} \cup B_{3}$, or $B_{2} \cup B_{3} \cup B_{4}$, or $B_{3} \cup B_{4} \cup B_{1}$, or $B_{4} \cup B_{1} \cup B_{2}$.
} 
$\operatorname{Div}_{c}\left(b_{t}, b_{r},\left(b_{t}, c\right)\right) \cup\left\{\left(b_{r}, c\right)\right\}$. Suppose that $\left(b_{r}, c\right)$ has one interior edge $f$ properly incident to it. By B2 and B5, $f$ is is incident to a blue node $b_{x}$ in $\left[b_{r}, b_{t}\right]$. Thus, the candidate tree equals $G a p\left(b_{r}, b_{x}\right) \cup \operatorname{Div}_{c}\left(b_{t}, b_{x},\left(b_{t}, c\right)\right) \cup\left\{f,\left(b_{r}, c\right)\right\}$ if the position of $b_{x}$ is known. We try all $O(k)$ choices for $b_{x}$ to find the position that minimizes the total length. The candidate trees for the two cases where $\left(b_{t}, c\right)$ has at most one interior edge properly incident to it can be handled similarly.

Case (1.2): $b_{r}$ and $b_{t}$ lie on the same boundary staircase. W.l.o.g., assume that $b_{r}$ and $b_{t}$ lie on $B_{4}$. See Figure $9(\mathrm{~b})$. As in case (1), the subtree that connects $S\left[b_{t}, b_{r}\right]$ and the complete interior corner equals the minimal $C\left(b_{t}, a_{i},\left(b_{t}, c\right)\right) \cup$ $C_{c}\left(b_{r}, a_{i+1},\left(b_{r}, c\right)\right)$ for some neighboring points $a_{i}, a_{i+1} \in S\left[b_{t}, b_{r}\right]$. By B3 and B4, there must be exactly one interior edge properly incident to $\left(b_{r}, c\right)$ or $\left(b_{t}, c\right)$ so that we cannot flip the complete interior corner to overlap with $B_{4}$. By $\mathrm{B} 2$ and $\mathrm{B} 5$, such an interior edge is incident to a blue node in $\left[b_{r}, b_{t}\right]$. Suppose that such an interior edge is properly incident to $\left(b_{r}, c\right)$ and a blue node $b_{x}$. Then the subtree that connects $S\left[b_{r}, b_{t}\right]$ and the complete interior corner equals $G a p\left(b_{r}, b_{x}\right) \cup \operatorname{Div}_{c}\left(b_{t}, b_{x},\left(b_{t}, c\right)\right) \cup\left\{\left(b_{r}, c\right)\right\}$. (Note that $\left[b_{x}, b_{t}\right]$ may not lie on three boundary staircases and in such cases, $\operatorname{Div}_{c}\left(b_{t}, b_{x},\left(b_{t}, c\right)\right)$ is an extra-division.) Thus, it suffices to try all $O(k)$ positions for $b_{x}$ to find the position that minimizes the total length.

Case (1.3): $b_{r}$ and $b_{t}$ lie on consecutive boundary staircases. For example, $b_{r}$ lies on $B_{3}$ and $b_{t}$ lies on $B_{4}$. This case can be handled by a method similar to the one in case (2).

The minimal Group-3 tree contains a complete interior line. For each grid line $\left(b_{r}, b_{t}\right)$, we compute a minimal tree for the case that $\left(b_{r}, b_{t}\right)$ is contained in the minimal Group- 3 tree. There are $O(n)$ grid lines and the minimal tree among all the $O(n)$ cases is the desired $G_{3}$. For each blue (resp. non-blue) grid line, we show how to compute a minimal tree in $O(n)$ time (resp. $O(1)$ time). Thus, the total processing time is $O(k n)$. When $\left(b_{r}, b_{t}\right)$ is blue, there are three cases as listed below:

Case (2.1): $b_{r}$ and $b_{t}$ lie on opposite boundary staircases. The minimal tree is partitioned along $\left(b_{r}, b_{t}\right)$ into two divisions: $\operatorname{Div}\left(b_{r}, b_{t},\left(b_{r}, b_{t}\right)\right) \cup \operatorname{Div}_{e}\left(b_{r}, b_{t},\left(b_{r}, b_{t}\right)\right)$. See Figure 9(e). This takes $O(1)$ time to construct.

Case (2.2): $b_{r}$ and $b_{t}$ lie on two consecutive boundary staircases and there exists an interior edge that is properly incident to $\left(b_{r}, b_{t}\right)$ and intersects a boundary staircase different from the two containing $b_{r}$ and $b_{t}$. W.l.o.g., assume that the configuration looks like Figure $9(\mathrm{c})$. Among all the interior edges that are properly incident to $\left(b_{r}, b_{t}\right)$ and intersect a boundary staircase different from the two containing $b_{r}$ and $b_{t}$, let the farthest one from $b_{r}$ be incident to an exterior vertex $b_{x}$ in $\left[b_{t}, b_{r}\right]$. If the position of $b_{x}$ is known, then the tree in Figure $9(\mathrm{c})$ equals $\operatorname{Div}\left(b_{r}, b_{t},\left(b_{r}, b_{t}\right)\right) \cup$ $C_{c}\left(b_{r}, b_{x},\left(b_{r}, b_{t}\right)\right) \cup G a p\left(b_{t}, b_{x}\right)$. We try all $O(n)$ positions for $b_{x}$ to find the one that minimizes the total length.

Case (2.3): $b_{r}$ and $b_{t}$ lie on two consecutive boundary and there is no interior edge that is properly incident to $\left(b_{r}, b_{t}\right)$ and intersects a boundary staircase different from the two containing $b_{r}$ and $b_{t}$. W.l.o.g., assume that the configuration looks like Figure 9(d). By rotational symmetry, we can assume that there is a longest complete interior line which is vertical. Thus, we can further assume that $\left(b_{r}, b_{t}\right)$ is a longest complete interior line and it is the rightmost one among all longest vertical complete interior lines. The reason is that such an assumption will definitely satisfy for some choice of $\left(b_{r}, b_{t}\right)$ as we are exhausting all possible choices. Imagine that a carpet is unrolled starting from $\left(b_{r}, b_{t}\right)$ to the right until the carpet touches the boundary. The carpet covers a rectangular area and we denote it by $R$ in Figure 9(d). We claim that the interior of $R$ is empty. Assume to the contrary that the interior of $R$ is non-empty. By definition of Group-3, any interior line intersecting $R$ cannot be a leg of an interior corner. Also, we can assume that any interior line intersecting $R$ is not the body of a $\mathrm{T}$; otherwise the $\mathrm{T}$ will produce the configuration in in Figure 9(c) which have already been handled. Thus, any interior line intersecting $R$ is a complete interior line and so it must either be longer than $\left(b_{r}, b_{t}\right)$ or has the same length and lies to the right of $\left(b_{r}, b_{t}\right)$. This contradicts our assumption and so $R$ must be empty. Let $b_{x}$ other than $b_{r}$ and $b_{t}$ be an exterior vertex at a corner of $R$ as shown in Figure $9(\mathrm{~d})$. If $b_{x}$ is a terminal point, then a candidate tree is the minimal of Tree $\left(b_{r}, b_{t}\right) \cup \operatorname{Gap}\left(b_{t}, b_{x}\right) \cup \operatorname{Tree}\left(b_{x}, b_{r}\right)$ and Tree $\left(b_{r}, b_{t}\right) \cup \operatorname{Tree}\left(b_{t}, b_{x}\right) \cup G a p\left(b_{x}, b_{r}\right)$. If $b_{x}$ is not a terminal point, then we also need to compare the above two candidates with $\operatorname{Tree}\left(b_{r}, b_{t}\right) \cup \operatorname{Tree}\left(b_{t}, a_{i}\right) \cup \operatorname{Tree}\left(a_{i+1}, b_{r}\right)$, where $a_{i}$ and $a_{i+1}$ are the two terminal points in $S\left[b_{t}, b_{r}\right]$ neighboring to $b_{x}$, in order to select the minimal one. Note that $b_{x}$ is known for a given $\left(b_{r}, b_{t}\right)$. Thus, the above processing takes $O(1)$ time.

This completes the discussion for the case where $\left(b_{r}, b_{t}\right)$ is blue. When $\left(b_{r}, b_{t}\right)$ is non-blue, then by $\mathrm{B} 6,\left(b_{r}, b_{t}\right)$ cannot contain any Steiner point. This makes the configuration similar to Figure $9(\mathrm{~d})$. In fact, the same analysis can be carried over to construct a minimal tree for this case. Thus, the case where $\left(b_{r}, b_{t}\right)$ can be handled in $O(1)$ time. 
Consider Figure 7(b). Recall that $\left[b_{r}, b_{t}\right]$ is assumed to lie on $B_{1} \cup B_{2} \cup B_{3}$ and $\left[b_{r}, b_{t}\right]=\left\{b_{r}, b_{r+1}, \cdots, b_{t-1}, b_{t}\right\}$. Let $f$ be the interior edge properly incident to $\left(b_{r}, v\right)$. Let $b_{z}$ be the exterior vertex in $\left[b_{r}, b_{t}\right]$ such that $\left(b_{z}, b_{t}\right)$ is a horizontal grid line. Let $b_{p}$ be last node in the sequence $\left[b_{r}, b_{z}\right]$ (recall that a node is a terminal point or an inner boundary corner vertex). Let $b_{q}$ be the lower endpoint of the vertical boundary edge that contains $b_{z}$. Let $a_{p}\left(a_{q}\right)$ be the terminal point closest to $b_{p}\left(b_{q}\right)$ in $S\left[b_{p}, b_{x}\right]\left(S\left[b_{q}, b_{x}\right]\right)$. Let $g$ be the interior edge immediately below $f$ and properly incident to $(v, c)$.

Lemma B.1 (Lemma 7.2) Let $T$ be a tree of configuration $C$ such that $T$ cannot be converted to a Class- 1 tree or a tree of configuration $A$ by sliding edges and flipping corners. If the total length of $T$ equals that of $T r e e\left(b_{r}, b_{t}\right)$, then one of the following holds:

1. $f$ can be slid downward until it contains $b_{p}$, and after the sliding, $b_{p}\left(a_{p}\right)$ is connected by a tree path in $T$ to $\left(b_{r}, v\right)((v, c))$ that uses $f(g)$,

2. $b_{q}$ is a terminal point, $f$ can be slid downward until it contains $b_{z}$, and $b_{q}\left(a_{q}\right)$ is connected by a tree path in $T$ to $\left(b_{r}, v\right)((v, c))$ that uses $f(g)$.

Proof Let the interior edge $f$ be incident to a tree edge $h$ that lies on the boundary. If $f$ is incident to two tree edges, then let $h$ be the vertical one. We prove several facts from which the lemma will follow.

1. Let $b_{m}$ be the exterior vertex at the intersection $f \cap h$. Then $B\left(b_{r}, b_{m}\right)$ does not monotonically decrease in both $x$ and $y$ coordinates and this implies that $B\left(b_{m}, b_{x}\right)$ lies on $B_{1} \cup B_{2}$. Let $b_{l}$ be the blue terminal in $\left[b_{r}, b_{m}\right]$ closest to $b_{r}$. The tree path connecting $b_{l}$ to $\left(b_{r}, c\right)$ cannot pass through $b_{r}$ and so the path must use $f$. (Otherwise, the tree path lies on $B\left(b_{r}, b_{l}\right)$ as $B\left(b_{r}, b_{l}\right)$ is a shortest path between $b_{r}$ and $b_{l}$. This implies that we can slide $\left(b_{r}, c\right)$ to the left to become incident to blue exterior vertex in $\left[b_{r}, b_{m}\right]$ closest to $b_{r}$. But then the resulting tree is in Class-1 which contradicts our assumption.) We claim that $B\left(b_{r}, b_{m}\right)$ does not monotonically decrease in both $x$ and $y$ coordinates and this implies that $B\left(b_{m}, b_{x}\right)$ lies on $B_{1} \cup B_{2}$. Suppose that $B\left(b_{r}, b_{m}\right)$ monotonically decreases in both $x$ and $y$ coordinates. Since $b_{l}$ is connected by a tree path to $\left(b_{r}, c\right)$ that uses $f$, we can slide $f$ upward until it overlaps with the boundary. Since $f$ cannot overlap with any tree edge in the sliding, $f$ must be incident to $b_{l}$ and $b_{r}$ after the sliding. But the resulting tree is of configuration A which contradicts our assumption.

2. If $h$ is horizontal, then let $b_{h}$ be $b_{m}$. If $h$ is vertical, then let $b_{h}$ be the lower endpoint of the maximal sequence of vertical and adjacent tree edges containing $h$. Let $a_{h}$ be the terminal point closest to $b_{h}$ in $S\left[b_{h}, b_{x}\right]$. Then $a_{h}$ is connected by a tree path to $\left(b_{r}, c\right)$ that uses $g$. Assume to the contrary that the tree path connecting $a_{h}$ to $\left(b_{r}, c\right)$ uses $f$. Then this tree path contains $B\left(b_{h}, b_{h+1}\right)$ as $B\left(b_{h}, b_{h+1}\right)$ is a shortest path between $b_{h}$ and $a_{h}$. If $h$ is horizontal, then $B\left(b_{h}, b_{h+1}\right)$ is contained in a vertical tree edge. But then by our way of choosing $h$, we would have chosen this vertical tree edge as $h$ instead, a contradiction. If $h$ is vertical, then $B\left(b_{h}, b_{h+1}\right)$ is horizontal. We slide $f$ downward. Since $B\left(b_{m}, b_{x}\right)$ lies on $B_{1} \cup B_{2}, f$ will overlap with $B\left(b_{h}, b_{h+1}\right)$ and the total length will be reduced, a contradiction.

3. Suppose that $b_{h}$ is not below $v$. If we slide $f$ downward until $f$ contains $b_{h}$, then $f$ also contains the last node in the sequence $\left[b_{r}, b_{z}\right]$. Slide $f$ downward until it contains $b_{h}$. If the right endpoint of $f$ is $v$, then we are done. Suppose not. Then $b_{h}$ is strictly above $v$. Let the right endpoint of $f$ on $\left(b_{r}, c\right)$ be $u$. There are two cases: (1) $b_{h}$ and $a_{h}$ are contained in the same boundary edge, $(2) B\left(b_{h}, a_{h}\right)$ contains one inner boundary corner vertex. In case (1), we claim that $a_{h}$ must lie below $v$. Otherwise, we can add $B\left(b_{h}, a_{h}\right)$ to create a cycle and remove the edge joining $u$ and $v$ to break the cycle. Note that the total length does not increase. $u$ becomes the vertex at a newly created complete interior corner. We flip this corner to overlap with the boundary edge containing $b_{r}$. The overlapping is a line segment and we denote it by $A$. Since $B\left(b_{r}, b_{m}\right)$ does not decrease monotonically in both $x$ and $y$ coordinates, there is an inner boundary corner vertex directly above $b_{h}$. The vertical grid line, say $L$, incident to this inner boundary corner vertex is blue by definition. Thus, the upper endpoint of $L$ is blue and it lies on $A$. Hence, after the flipping, the tree is in Class- 1 which contradicts our assumption. In case (2), we can similarly prove that $a_{h}$ must lie below $v$ and the inner boundary corner vertex contained in $B\left(b_{h}, a_{h}\right)$ is the last node in the sequence $\left[b_{r}, b_{z}\right]$. Note that $f$ contains this inner boundary corner vertex.

4. If $b_{h}$ is below $v$, then $b_{h}$ is an outer boundary corner vertex and so it must be a terminal point. Assume to the contrary that $b_{h}$ is not an outer boundary corner vertex. Then $b_{h}$ and $a_{h}$ must be contained in the same boundary edge. Slide $f$ downward until it contains $b_{h}$. $f$ cannot contain $a_{h}$; otherwise, a cycle will be created. 
segment. The fact that $f$ can be slid along $B\left(b_{m}, a_{h}\right)$ implies that $B\left(b_{m}, a_{h}\right)$ is contained in the tree. Let $u$ be the right endpoint of $f$ on $\left(b_{r}, c\right)$ after the sliding. We add $B\left(b_{h}, a_{h}\right)$ to create a cycle and then break the cycle by removing the edge on $\left(b_{r}, c\right)$ incident to $u$ and the right endpoint of $g$. Note that the total length does not increase. $u$ will become the vertex at a newly created complete interior corner. But then this complete interior corner can be flipped to overlap with $B\left(b_{m}, a_{h}\right)$ to reduce the total length. This is a contradiction.

Now, if $b_{h}$ is not below $v$, then we can slide $f$ downward as stated in fact 3 , and $b_{p}$ is the last node in the sequence $\left[b_{r}, b_{z}\right]$ contained by $f$. If $b_{h}$ is below $v$, then $b_{q}=b_{h}$ and $f$ can be slid downward until it contains $b_{z}$. We have $a_{p}=a_{h}$ in the first case and $a_{q}=a_{h}$ in the second case. 